\title{
Review on adaptive control of laser-directed energy deposition
}

\author{
Hao Wang, ${ }^{a}$ Weiwei Liu, ${ }^{\text {a,* }}$ Zijue Tang, ${ }^{a}$ Yiwen Wang, ${ }^{\text {a }}$ Xiaolei Mei, ${ }^{a}$ \\ Kazi M. Saleheen, ${ }^{a}$ Zhenqiu Wang, ${ }^{a}$ and Hongchao Zhang ${ }^{b}$ \\ ${ }^{a}$ Dalian University of Technology, School of Mechanical Engineering, Dalian, China \\ ${ }^{\mathrm{b}}$ Texas Tech University, Department of Industrial, Manufacturing, and Systems Engineering, \\ Lubbock, Texas, United States
}

\begin{abstract}
Laser directed energy deposition (LDED) is one of the most important parts of metal additive manufacturing, which can provide fast building speed, allows for large building volumes, and is suitable for part repair. LDED can manufacture components layer by layer through processes of rapid heating, melting, solidification, and cooling with the laser beam as a heat source. However, deposition quality and repeatability of components produced by LDED are poor because of the complex thermal cycle and processing environment, hindering the spread of this technique. Adaptive control technology (ACT) is consistently considered an effective and potential way to solve the problem. Many studies have focused on LDED and established the relations of process parameters, process signatures, and product qualities, which promote the rapid development of ACT, with the development of monitoring devices and data processing technology. We review and discuss the problems existing in the ACT of LDED. (c) 2020 Society of Photo-Optical Instrumentation Engineers (SPIE) [DOI: 10.1117/1.OE.59.7.070901]
\end{abstract}

Keywords: laser directed energy deposition; additive manufacturing; process monitoring; quality prediction; adaptive control.

Paper 20200259V received Mar. 3, 2020; accepted for publication Jun. 23, 2020; published online Jul. 6, 2020.

\section{Introduction}

Additive manufacturing (AM), a technique that can manufacture a part layer by layer, is contrary to traditional subtractive manufacturing (e.g., machining and milling). ${ }^{1}$ It has the advantages of faster processing speed, lower manufacturing cost, and lower energy consumption, and can produce more complex parts compared with other traditional manufacturing methods. In the fields of aerospace, automobile, biomedicine, and remanufacturing, AM is considered an important manufacturing method. One important field of AM is the production of functional metal components with complex shapes, including metals, alloys, and metal matrix composites. ${ }^{2}$ Laser directed energy deposition (LDED) is one of the most commonly used techniques in the AM of metal. Figure 1 shows that a high-power laser beam is used as the heat source and feedstocks are sent into the molten pool in the form of powders or wires in LDED. ${ }^{3}$ Moreover, the substrate and materials are melted with the movement of a laser beam, then the part will be manufactured layer by layer after the solidification of the molten pool. LDED enables generating custom parts with complex shapes and functional gradients, demonstrates the ability to revolutionize manufacturing, and has broad prospects in direct shaping, surface coating, and remanufacturing repair. ${ }^{4,5}$

However, several problems, including low quality of layers, difficult and expensive postprocess inspection, and accumulative errors, exist in LDED. Adaptive control technology (ACT) has been widely used in LDED and reviewed by several scholars. ${ }^{5,6}$ Figure 2 shows that ACT includes monitoring of the process, prediction of the quality based on relations of the parameters, signatures, and qualities, and adaptive control of the final quality by adjusting the parameters. Most of the relevant pieces of literature are not comprehensive and systematic, and the relations

*Address all correspondence to Weiwei Liu, E-mail: liuww@dlut.edu.cn 


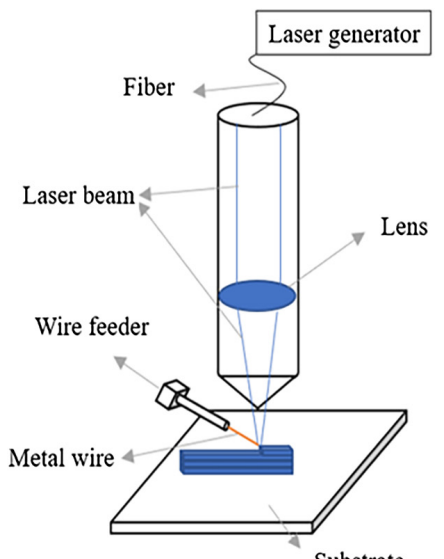

(a)

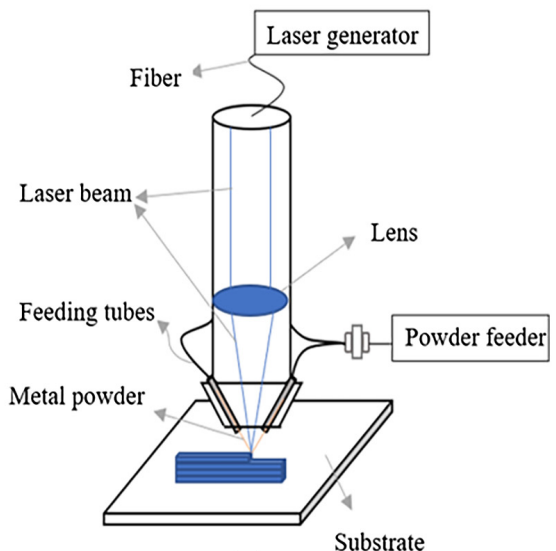

(b)

Fig. 1 Schematic diagram of LDED: (a) wire feeding and (b) powder feeding.

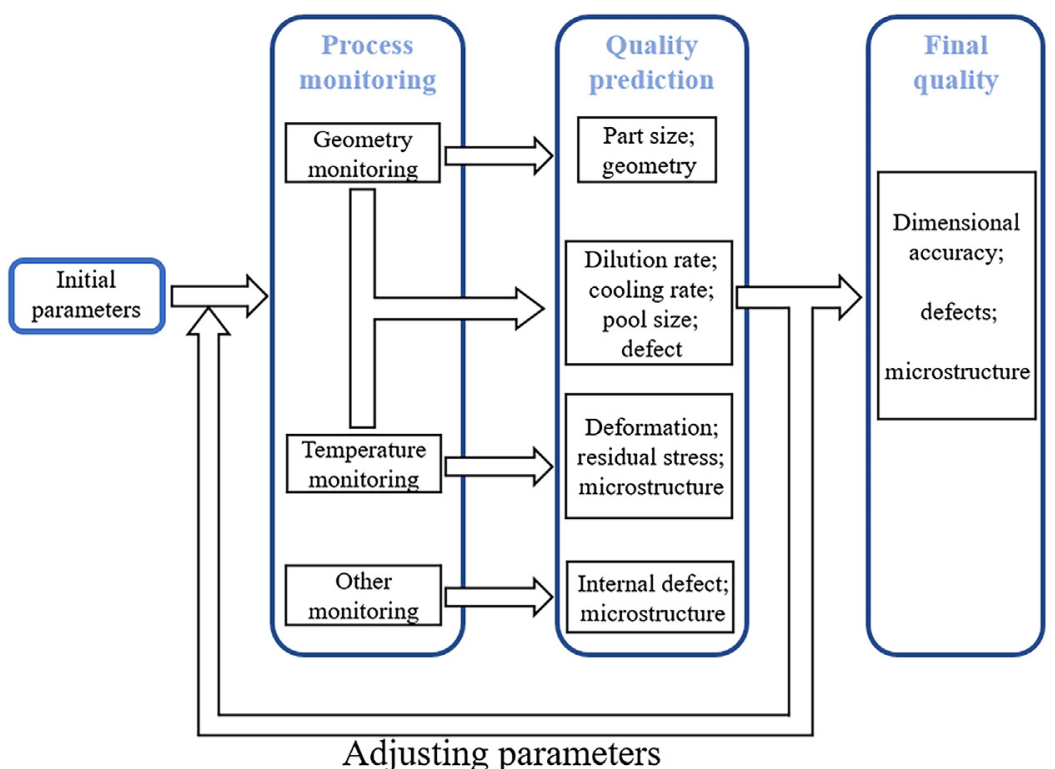

Fig. 2 Main process diagram of the adaptive control of LDED.

of process parameters, signatures, and product qualities are rarely reviewed. This paper tries to comprehensively review the research progress of the ACT in LDED from process monitoring (Sec. 2), relations of parameters, signatures, qualities (Sec. 3), and adaptive control methods (Sec. 4). In addition, the discussion (Sec. 5) and conclusion (Sec. 6) are given.

\section{Process Monitoring}

Process monitoring has become a research hotspot to obtain high quality and repeatability of LDED parts. Generally, monitored signatures include geometry and temperature. Vision sensors and temperature sensors are widely used in monitoring LDED.

\subsection{Geometry Monitoring}

\subsubsection{Devices and basic principles}

In LDED, high-speed cameras and three-dimensional (3-D) scanners are used to monitor geometry signatures. Charge-coupled device (CCD) cameras $^{7,8}$ and complementary metal oxide 
semiconductor (CMOS) cameras ${ }^{9,10}$ are two commonly used image acquisition devices. According to experimental requirements and the actual situation on site, the position of the camera can be divided into coaxial ${ }^{9,11}$ and off-axis. ${ }^{12}$ Coaxial monitoring can track the molten pool movement in real time and record the dynamic change of the molten pool, whereas offaxis monitoring can capture the information of the whole processing scene and avoid the interference caused by the vibration of the laser head. Despite frequent use of high-speed cameras in LDED, it is mainly used for process monitoring and analysis instead of ACT due to limited processing speed. Moreover, the 3-D camera is a combination of monocular or binocular vision and structural light to monitor the entire shape of the deposited layer. The 3-D camera can only be installed at a fixed point, which is a limitation for ACT. Another device applied to geometry monitoring is the high-dynamic camera, which is suitable for monitoring the dynamic molten pool and other situations with a large brightness span. Although imaging quality is not very high, the frame rate of $50 \mathrm{fps}$ with a high-dynamic camera can meet the requirements of ACT.

Lighting devices, such as light-emitting diodes, laser diodes, and vertical cavity surface emitting lasers, ${ }^{13,14}$ are also needed for more accurate visual information. A filter must be used to eliminate the interference of energy beam radiation and powder and obtain the best image of the molten pool. ${ }^{7,9,10}$ Other related devices will also be described below.

\subsubsection{Geometry signature monitoring}

At present, many researchers have monitored geometry signatures using different devices and algorithms in LDED. Geometry signatures include the outline of the deposited layers and the profile of the molten pool. In these geometry signatures, the widths and heights of the deposited layers ${ }^{15}$ and the profile of the molten pool ${ }^{9,10}$ can be monitored by a single industrial camera, and the entire outline of the deposited layers are commonly obtained by a 3-D camera. ${ }^{16,17}$ To improve the calculation speed and efficiency, Tang et al. ${ }^{18}$ used a laser-based areal topography measurement sensor to measure the surface and developed a depth image method to process the data and extract the 3-D features. Moreover, a 3-D scanner of structural light was used to measure the height of the deposited layers from an external fixed position by Garmendia et al. ${ }^{17}$ However, the strong metal vapor in the 3-D reconstruction method based on structural light may contaminate the optical device and affect the monitoring results. A new method to extract the 3-D structure based on the position information of the electron beam speckle was proposed to overcome the deficiency of structural light monitoring. ${ }^{19}$ In this method, the electron beam is used to bombard the surface of the part to produce a thermal effect and form speckles, and the 3-D reconstruction will be achieved based on the structural information of the electron beam in the vacuum chamber.

During manufacturing, distortion of parts will greatly influence the final geometric accuracy of LDED. Scholars commonly use displacement sensors for in-situ monitoring of substrate distortion. In recent years, digital image correlation (DIC) technology has also been used to monitor the distortion of LDED. DIC uses binocular stereo vision technology and tracks the speckle image of the object's surface; geometric point displacement is obtained using the correlation algorithm. Studies such as that conducted by Ocelik et al..$^{20}$ have shown that DIC could measure the in-situ strain occurring on the substrate during metal deposition, whereas other studies used this technology to monitor surface distortion of the deposited layers. ${ }^{21-23}$

In summary, monitoring geometry signatures have high requirements on sensing equipment, and advanced equipment is the foundation of obtaining accurate process signatures and product qualities. A fast, reliable image processing algorithm is also a research focus to enhance monitoring ability.

\subsection{Temperature Monitoring}

Temperature signatures of LDED are directly related to product quality, and temperature monitoring is important for the ACT of LDED. Figures 3 and 4 show that LDED is a cycle of rapid heating and cooling, which makes monitoring difficult. Therefore, the monitoring sensor needs high-speed data acquisition and high resolution to capture the temperature signatures in LDED. ${ }^{26}$ 


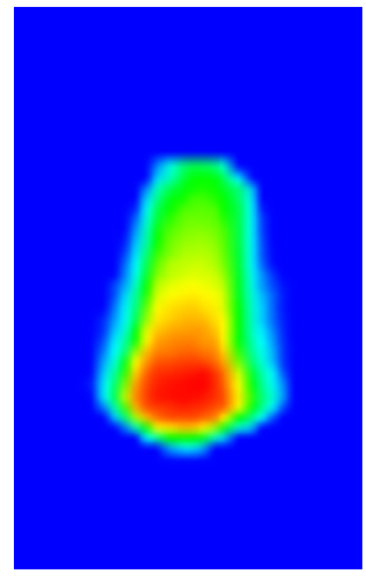

(a)

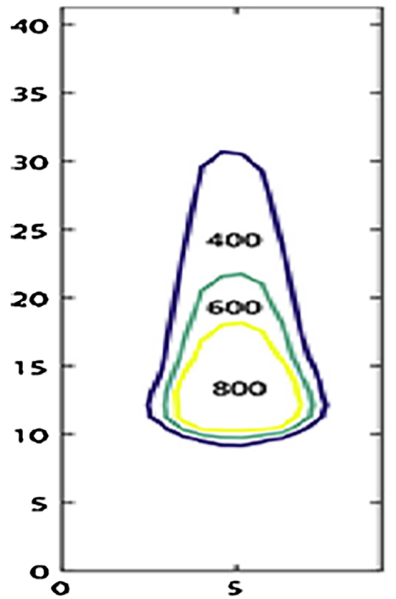

(b)

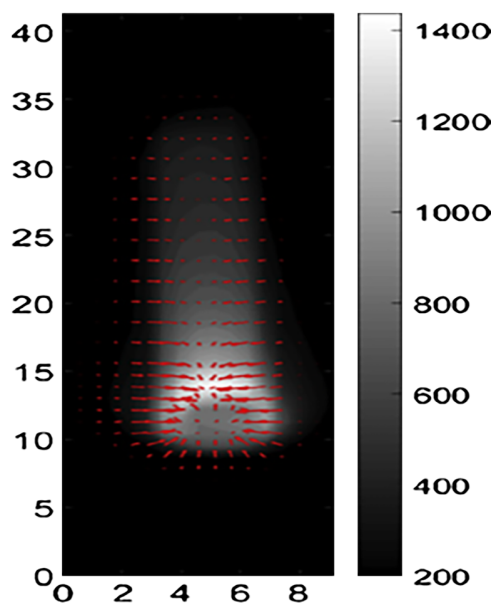

(c)

Fig. 3 (a) IR image, (b) isotherm, and (c) surface temperature gradient of molten pool in LDED. ${ }^{24}$

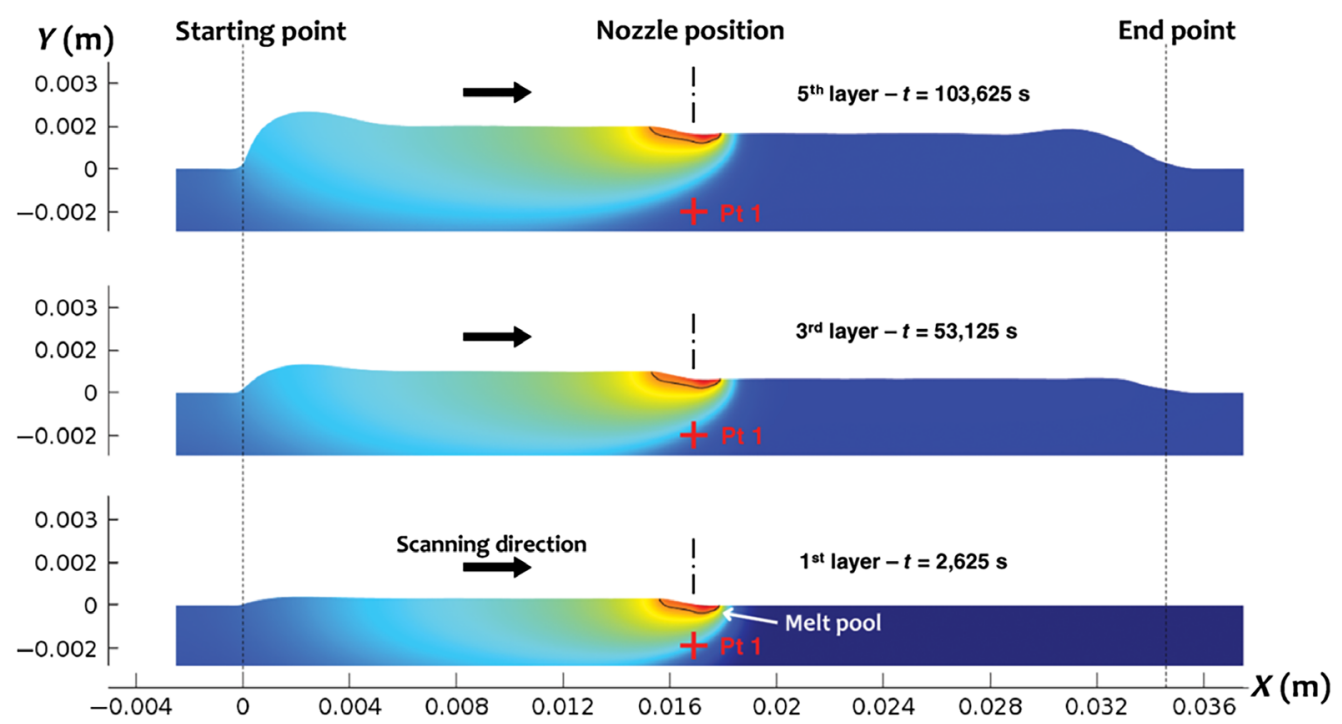

Fig. 4 Model of temperature field and pool shape for the first, third, and fifth layers. ${ }^{25}$

\subsubsection{Devices and basic principles}

Methods of collecting temperature signals include contact measurement and noncontact measurement.

Contact measurement is generally monitored by a thermocouple, which consists of two metal wires of different materials to form a closed loop. The temperature gradients at both ends generate a thermoelectric electromotive force and convert the temperature signal into an electrical signal. A thermocouple has the advantages of low cost and high accuracy, but its application in LDED is limited by the dynamic molten pool, high temperature, and high-energy beam interference. Thus, it is used for monitoring substrate temperature, such as welding thermocouples to the underside of the substrate to record the thermal cycle during deposition. ${ }^{24,27}$ Moreover, several measures, such as using protective sheets, are necessary to decrease the risk of destroying the thermocouple wires. ${ }^{28}$

Noncontact measurement is based on the principle that the infrared (IR) radiation of an object changes with temperature. According to Planck's law, the radiation of a blackbody is ${ }^{29}$

$$
L\left(T_{B}, \lambda\right)=\frac{C_{1}}{\lambda^{5}\left[e^{C_{2} /\left(\lambda T_{B}\right)}-1\right]},
$$


where $C_{1}=2 h c^{2}, C_{2}=\frac{h c}{k}, L$ is the radiation of the blackbody, $h$ is the Planck's constant, $k$ is the Boltzmann constant, $c$ is the velocity of light, $\lambda$ is the wavelength, and $T$ is the absolute temperature. The radiation of an object can be expressed as the product of a blackbody's radiation and the emissivity $\varepsilon$.

$$
L_{r}\left(T_{B}, \lambda\right)=\varepsilon L\left(T_{B}, \lambda\right)=\varepsilon \frac{C_{1}}{\lambda^{5}\left(e^{\frac{C_{2}}{T_{B}}}-1\right)}
$$

The absolute temperature of an object can be restored based on the radiation data acquired by sensors and the wavelengths. Compared with a thermocouple, IR sensors neither need to directly contact the objects nor affect temperature distribution. The short response time and the ability to monitor the dynamic pool make IR temperature measurement widely adopted in LDED.

In addition to commonly used IR equipment, a spectrometer is a noncontact measurement instrument for temperature. Several studies ${ }^{30-32}$ used the spectral information within the emitted light of the molten pool to form a key enabling element in the estimation of emissivity and to reveal the temperature information.

\subsubsection{Temperature signature monitoring}

Objects of temperature monitoring are classified into temperature change of a single point, temperature distribution of the molten pool, and temperature distribution of the deposited layers. In the current studies, a thermocouple was mainly used for the single-point temperature of the substrate, whereas more studies used IR devices to monitor temperature signatures. For example, Bi et al. ${ }^{33,34}$ detected the IR radiation flux of the molten pool with a germanium photodiode and converted it into an IR temperature signal. Moreover, several studies used the monochromatic pyrometer to record the thermal cycle of deposition. ${ }^{35-37}$ However, the calibration of devices is a limitation of IR temperature measurement because determining emissivity is difficult and further complicated by physical transformations that the material undergoes during processing. ${ }^{6}$ Measurement with a two-color pyrometer ${ }^{15,38-41}$ is an effective method to avoid unknown emissivity and particle interference and to obtain an accurate value of temperature. Because the emissivities of an object with two close wavelengths are approximately equal, according to Eq. (2), the ratio of radiation energies in two wavelengths will reduce the effect of emissivity. Moreover, Pavlov et al. ${ }^{42}$ and Doubenskaia et al. ${ }^{43}$ monitored the temperature of the molten pool for a more stable signal with a multicolor pyrometer. Temperature distribution is usually monitored by IR imaging equipment. For example, Yang et al. ${ }^{44}$ used an IR camera to collect the surface temperature of the deposited parts and studied the thermal behavior. Farshidianfar et al. ${ }^{45,46}$ used IR cameras and image processing technology to capture the temperature changes and cooling rates in real time.

The application of multiple devices can obtain more comprehensive temperature signatures because of the different monitoring objects of various devices. Smurov et al. ${ }^{47}$ comprehensively analyzed deposition by monochromatic pyrometer, multiwavelength pyrometer, and IR camera, and presented a fusion method of diverse temperature data. In the research conducted by Liu et al., ${ }^{48}$ the variation of the molten pool temperature was measured by a pyrometer; temperature distribution, molten pool size, and cooling rate were studied by an IR camera.

According to the current research, temperature monitoring is easily affected by the environment. Several measures must be taken to avoid interference caused by the complex environment and ensure data accuracy. Emissivity is an important factor affecting accuracy of noncontact measurements. Thus, determination of emissivity and multidevice coupling are also effective methods for improving accuracy. Table 1 summarizes devices used for temperature monitoring in different references.

\subsection{Other Monitoring}

Several researchers also monitored other signatures that may affect the final qualities, such as detection of particle-in-flight velocity with a diagnostic tool based on CCD camera ${ }^{47}$ and 
Wang et al.: Review on adaptive control of laser-directed energy deposition

Table 1 Devices for temperature monitoring.

\begin{tabular}{lll}
\hline \hline Monitoring signatures & \multicolumn{1}{c}{ Devices } & \multicolumn{1}{c}{ References } \\
\hline Temperature of substrate & Thermocouple & 24,27 , and 28 \\
Temperature of molten pool & Monochromatic pyrometer & $33,36,37$, and 47 \\
& Two-color pyrometer & $15,38-41$, and 48-50 \\
& Multicolor pyrometer & 42 and 43 \\
& Hyperspectral & $30-32$ \\
Temperature distribution of deposited layers & Photodiode & 33,34 , and 51 \\
Temperature distribution of molten pool & 2-D pyrometer & $24,44,47,49$, and 50 \\
\hline \hline
\end{tabular}

monitoring of powder flow using a photoelectric sensor. ${ }^{52}$ In general, cracks and pores are important symbols of defects in LDED and are difficult to monitor. The earliest attempt was to monitor cracks using acoustic emission sensors, ${ }^{53,54}$ but it was easily affected by external interference in field monitoring. Vargas et al. ${ }^{55}$ studied the scaling of the x-ray fluence and energy with electron beam properties and laser power. The high-contrast imaging of various test objects clearly showed the defects in AM, and the possibility of field measurement of AM in the future was discussed. Wolff et al. ${ }^{56}$ performed field high-speed x-ray imaging of individual powder particles flowing into the molten pool, and the effect of beam-matter interaction on flow and pore formation of powder was revealed. In addition, Sharples et al. ${ }^{57}$ developed a spatially resolved acoustic spectroscopy (SRAS) system, which is a laser ultrasound inspection technique for imaging the material microstructure of metals. SRAS uses a pulse laser to generate acoustic waves on a surface and measures surface disturbances using another laser and a detector. This technique is also sensitive to surface and subsurface pores, and has been used to identify and classify surface defects and pores in AM. ${ }^{58-60}$

In LDED, several key elements can define the properties of the deposited parts. ${ }^{61}$ At present, $\mathrm{x}$-ray fluorescence and energy-dispersive spectroscopy have been widely used for element analysis, but neither method is suitable for online monitoring. Laser-induced breakdown spectroscopy (LIBS) has already been demonstrated as a powerful instrument for in-situ quantitative analysis and is suitable for high-speed monitoring in the industrial field. ${ }^{62}$ Recently, LIBS was also applied to AM monitoring. Song and Mazumder ${ }^{63}$ used LIBS to analyze real-time chromium composition in LDED. Moreover, Lednev et al.$^{61}$ developed a remote LIBS system to quantitatively analyze light (carbon and silicon) and heavy (tungsten and nickel) elements in LDED. This technique can provide good analytical results without affecting deposited qualities. In the future, online quantitative analysis in LDED will be essential to ensure the high quality of parts with a specifically designed gradient of elemental composition.

Traditional monitoring devices have been used in many studies and have allowed much progress in LDED. However, more advanced technologies must be applied in further research of LDED, which is very helpful for process understanding. The ACT of LDED will be greatly improved with more accurate, detailed process information.

\section{Relations of Parameters, Signatures, and Qualities}

LDED is a complex process with multiparameter interaction. Before adaptive control of product qualities, the relations of process parameters, process signatures, and product qualities must be determined. In the ACT of LDED, the deposited qualities are taken as a function of the parameters, and the qualities can be improved by changing the parameters according to the monitoring signatures. 


\subsection{Relations of Parameters, Geometry Signatures, and Qualities}

In LDED, geometry signatures, such as width and height of the deposited layers and morphology of the molten pool will affect the final geometric accuracy. Therefore, establishing the relations of parameters, geometry signatures, and qualities is crucial for adaptive control. The following is the related research. Ocelík et al. ${ }^{64}$ used a statistical analysis method to establish the process diagram between process parameters and cross-section geometric parameters and studied the relations of scanning speed, laser power, powder rate, and main geometry signatures of a single-laser track. Qi et al. ${ }^{65}$ proposed a more accurate quadratic regression transfer function to predict the width of the deposited layers and identified that the first three most important factors affecting wall thickness are laser power, scanning speed, and defocus distance. Another study observed that laser power is the main parameter that affects the geometry qualities of deposited layer; clad height, dilution, temperature, and stress all increased with the increase of laser power. ${ }^{66}$ Moreover, Sreeraj and Kannan ${ }^{67}$ reviewed the research progress of using neural network technology to establish cladding geometry prediction models and applied the basic idea of an artificial neural network to geometric modeling and prediction of composite materials.

Dilution rate is an important index, which is not only substantial for evaluating the quality, but also directly affects the interlayer bonding strength of as-formed parts. Figure 5 shows the cross section of deposited layers. Dilution can be calculated as follows: ${ }^{68}$

$$
\eta=\frac{A_{2}}{A_{1}+A_{2}},
$$

where $A_{1}$ is the cross-sectional area of deposited layers and $A_{2}$ represents the area of the molten zone of the substrate. The dilution of LDED is difficult to monitor and is generally calculated by models or other signatures of the molten pool. Hofman et al. ${ }^{69}$ established a model to determine the geometry qualities and dilution of LDED, and this model was used to study the correlation between signatures of the observable molten pool and dilution. Moreover, Xi et al. ${ }^{70}$ established a theoretical model to study the relation between power and dilution rate, and obtained the correlation coefficients of cladding area, fusion area, and dilution rate. This model can provide a basis for estimating dilution rate in LDED.

\subsection{Relations of Parameters, Temperature Signatures, and Qualities}

The complicated temperature field is one of the most important signatures of LDED and has an important effect on cooling rate and internal phase transformation. Much literature has considered thermal behavior in LDED. Bi et al. ${ }^{34}$ studied the influence of laser power, scanning speed, and feeding speed on molten pool temperature, and found that the dilution and size of cladding have a good correlation with the temperature signal. In addition, transient models are commonly used tools for thermal analysis and have been widely used in LDED, such as for the investigation of heat transfer in molten pools, ${ }^{71}$ analysis of solidification processes, and prediction of microstructure. For example, Huang et al. ${ }^{72}$ developed a thermal model to correlate the process parameters to the localized transient thermal signatures and solidification parameters and predict the microstructural evolution. Their conclusion can also be used for parameter optimization and in-situ microstructural control.

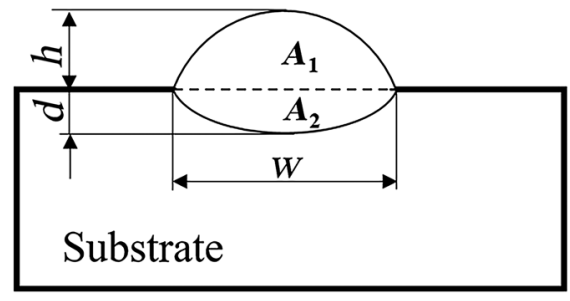

Fig. 5 The cross section of deposited layers. 
Residual stress and part distortion have become the bottleneck of LDED because of rapid thermal cycles in manufacturing. Many experiments and models were established by scholars to investigate the relationship between distortion and parameters. Denlinger et al. ${ }^{27}$ studied the effect of interlayer dwell time on thermal distortion and residual stress of the substrate. The experiment showed that adding dwell time to allow additional cooling could reduce the distortion and stress of Inconel 625, whereas the result of Ti-6Al-4V was the opposite. Mukherjee et al. ${ }^{73}$ used strain parameters and well-tested 3-D numerical models of heat transfer and fluid flow to reveal the effects of key process variables such as power and scanning speed on thermal strain. Then they used a coupled thermal, fluid flow, and a mechanical model to investigate the evolution of stresses and strains for Inconel 718 and Ti-6Al-4V and the relation of heat input and layer thickness. ${ }^{74}$ Moreover, Yan et al. ${ }^{24,26}$ summarized the typical thermal models, proposed the relations of process parameters, thermal behavior, and product quality, and expounded the influence of process parameters on thermal distortion of the workpiece. However, traditional numerical methods are limited by the long calculation time with the increase of the overall size of the component. Xie et al. ${ }^{75}$ proposed an efficient equivalent temperature field method to predict thermal distortion by extracting the quasisteady temperature field as the thermal boundary of mechanical analysis to solve this problem. This model greatly saved more than $90 \%$ of the calculation time in mechanical analysis and successfully predicted the distortion of 266-layers thin walls in a few hours.

For a generalization of the studies surveyed above, relations of parameters, signatures, and qualities are summarized in Table 2. In this table, quality is classified into geometric accuracy, defects, and microstructure with the comprehensive consideration of Ref. 5 and the actual process of manufacturing.

Table 2 Relations of parameters, signatures, and qualities.

\begin{tabular}{|c|c|c|c|}
\hline Qualities & Parameters & Signatures & References \\
\hline \multirow[t]{9}{*}{$\begin{array}{l}\text { Geometric } \\
\text { accuracy }\end{array}$} & $\begin{array}{l}\text { Scanning speed, laser power, } \\
\text { and powder feeding rate }\end{array}$ & Height, width, and dilution & 64 and $76-78$ \\
\hline & $\begin{array}{l}\text { Scanning speed, laser power, } \\
\text { and powder feeding rate }\end{array}$ & Pool shape & 79 \\
\hline & Laser power & Width & 65 \\
\hline & Laser power and scanning speed & Pool size & 34,80 , and 81 \\
\hline & Scanning speed and laser power & Dilution & 68 and 82 \\
\hline & $\begin{array}{l}\text { Laser power, height increment, } \\
\text { and cooling times }\end{array}$ & Width & 83 \\
\hline & Defocusing distance & Height & 84 \\
\hline & Dwell time & Temperature and distortion & 27 \\
\hline & $\begin{array}{l}\text { Laser power, scanning speed, } \\
\text { and substrate thickness }\end{array}$ & Temperature and distortion & 24 and $73-75$ \\
\hline \multirow[t]{2}{*}{ Defects } & Laser power and scanning speed & Cooling rate & 53 and 85 \\
\hline & $\begin{array}{l}\text { Laser power, scanning speed, } \\
\text { and feeding rate }\end{array}$ & Laser-matter interaction & 56 \\
\hline \multirow[t]{4}{*}{ Microstructure } & Dwell time & Molten pool size and cooling rate & 11 \\
\hline & Laser power & Temperature and pool size & 34 \\
\hline & Scanning speed and laser power & Temperature and cooling rate & 72 \\
\hline & Scanning speed and laser power & Temperature and cooling rate & 86 \\
\hline
\end{tabular}




\section{Adaptive Control Methods}

Based on data obtained from online monitoring and established relations, process parameters can be appropriately adjusted to realize adaptive control and ensure product quality. This section will review adaptive control methods from three views. According to control time, LDED includes online control and layer-to-layer control. From the view of the relation between monitoring signatures and concerning characteristics, LDED contains direct control and indirect control. From the classification of quality, LDED contains the control of geometric accuracy, defects, and microstructure.

\subsection{Online and Layer-to-Layer Control}

In online control of LDED, researchers need to adjust parameters based on real-time monitoring signatures to ensure that the signatures (height, width, and temperature) follow a reference curve such that the quality within the same deposited layer can be kept stable. Online control requires high-response speed to information; thus, scanning speed and laser power are usually selected as the parameters to be changed. Fathi et al. ${ }^{87}$ designed a proportional integral derivative (PID) feedforward controller to compensate the deviation of cladding height according to the relation between speed and height. Experiments showed that the feedforward PID controller could effectively control the process and reduce the response time and overshoot. In addition, Song and Mazumder. ${ }^{41}$ proposed a generalized predictive control strategy with constraints, which made the molten pool temperature follow the reference curve by adjusting the power. Hofman et al. ${ }^{9}$ obtained the width of the molten pool through a CMOS camera and adjusted the power through a feedback control system in real time to keep the width of the molten pool at the reference value.

Layer-to-layer control is realized by compensating the deviation of the previous layer. At the end of each layer, signatures of the entire deposited layer are extracted and compared with the expected results; then, the deviation can be compensated by modifying the scanning track and process parameters. Generally, the layer-to-layer control focuses on the control of height. For example, Garmendia et al. ${ }^{17}$ proposed a method to modify the number of layers to ensure accuracy of the deposition height and developed a new computer-aided manufacturing program that can calculate the subsequent scanning track according to the measured height and reduce the error between the actual value and the measured value. Moreover, Arrizubieta et al. ${ }^{88}$ developed a powder flow control system to regulate deposition height with the method of layer-to-layer control because the response speed of the powder feeding rate is slow and not suitable for online control. However, neglecting the dynamics that describe how the process evolves from layer to layer in modeling and process control can lead to dimensional instability because LDED is a two-dimensional (2-D) dynamic process. Sammons et al. ${ }^{89,90}$ proposed a 2-D model to describe the process dynamics quantitatively and qualitatively and designed a layer-to-layer controller with a theory of repetitive process control to improve LDED stability.

Several researchers have tried combining the two methods because it may not be able to compensate the deviation quickly only with online control or layer-to-layer control. Tang and Landers ${ }^{39,40}$ proposed an online molten pool temperature controller based on the empirical model, which can track the temperature reference of the single layer very well. Moreover, the layer-to-layer controller was established to adjust the power of the next layer using the iterative learning control method to ensure consistency of morphology in a multilayer deposition. Moreover, with a hollow laser beam ${ }^{91}$ that can optimize the distribution of laser intensity, Shi et al. ${ }^{92}$ manufactured parts with a variable width. At the end of each layer, the scanning speed of the next layer was adjusted to compensate the deposition height error according to the height of the previous layer. Another combination of two methods was conducted by Garmendia et al., ${ }^{16}$ who used a 3-D scanner based on structured laser light to measure the height of a part. The average height deviation between layers was highly corrected by recalculating the track to be deposited in the next layer, and the local height deviation within the layer was adjusted by simultaneously changing the scanning speed.

\subsection{Direct Control and Indirect Control}

In LDED, several characteristics, which are important to the qualities and can be monitored easily, such as temperature, height, or width of the deposited layers and shape of the molten 
pool, can be controlled directly based on monitoring signatures. Direct control can avoid the error caused by a complex transformation relation and make the process more intuitive and accurate. In several studies of direct control, only one input and one output were selected to reduce the complexity of the controller. Moralejo et al..$^{10}$ adjusted the laser power to control the final width based on the pool width monitored by CMOS cameras. Recently, the trend of direct control is from a single input, single output to multiple inputs, multiple outputs. Song et al. ${ }^{15}$ proposed a dual-input, single-output hybrid control system, including a master height controller and a slave temperature controller, to control the height of each deposited layer and the molten pool temperature. When the pool height exceeds the specified layer thickness, the main height controller prevents the control action of the temperature controller and reduces the laser power to avoid excessive accumulation. If the pool height is lower than the specified layer thickness, the temperature controller bypasses the height controller and dynamically adjusts the laser power to control the pool temperature. Cao et al. ${ }^{93-95}$ proposed a control-oriented multiple-input multipleoutput (MIMO) model. Laser power and scanning speed are taken as inputs to capture coupling dynamics, and deposition height and molten pool temperature are taken as outputs. Many different types of literature on direct control are available in Refs. 10, 52, and 96-99.

Unlike direct control, indirect control needs to establish the relationship between monitoring signatures and concerning characteristics. Several characteristics that are difficult to be monitored, such as dilution rate, are usually controlled by indirect control. Generally, dilution rate can be controlled by adjusting the width ${ }^{69}$ or temperature ${ }^{38}$ of the molten pool according to the calculation models. Chakraborty and Dutta ${ }^{82}$ proposed a simple linear regression model based on the energy balance method for dilution rate control. Moreover, the shape of the molten pool can be indirectly reflected by the specific isotherm according to Planck's law because the contour of the molten pool is determined by the melting point of materials. Ding et al. ${ }^{52}$ obtained the image of the molten pool using an IR imaging device and determined the isotherm corresponding to the contour of the molten pool in the IR image. A uniform pool size was obtained during LDED by adjusting the power. Furthermore, Devesse et al. ${ }^{31,32,100,101}$ proposed an isotherm migration method to track the isotherm of a workpiece and established a dynamic control system based on the physical model. Then, the width of the molten pool was controlled by adjusting the power according to the isotherm.

\subsection{Geometric Accuracy, Defects, and Microstructure Control}

The purposes of adaptive control include improving geometric accuracy, reducing defects, and improving microstructure. Generally, control of geometric accuracy is the most common, intuitive method, and much research has provided references. Qi et al. ${ }^{65}$ proposed an accurate quadratic regression transfer function to obtain a stable width by changing the laser power or scanning speed. Miyagi et al. ${ }^{51}$ developed an adaptive shape control system, which consists of a process monitoring system and a PID controller. In this system, the deposited shape is successfully controlled as required using the profile accuracy of the deposited layers. Moreover, distortion must be suppressed in the process to ensure the geometric accuracy of parts. In LDED, thermal stress caused by a complex temperature field is one of the most important factors leading to distortion, and many studies have been conducted to suppress distortion by decreasing the laser power or increasing the scanning speed. Heigel et al. ${ }^{102}$ proposed a new metric, cladding heat, which provides a new direction for distortion control. The formula for cladding heat is as follows: ${ }^{102}$

$$
\text { clad heat }=\frac{P h}{v},
$$

where $P$ is the laser power, $h$ is the overlap spacing, and $\nu$ is the scanning speed. In addition to laser power and scanning speed, they also considered the effect of overlap rate on the thermal distortion. Preheating and scanning paths are also effective methods to suppress distortion.

Normally, cracks and uneven pore distribution are common defects in LDED. If these defects cannot be effectively suppressed, they may lead to part failure or low part performance. Chen et al. ${ }^{85}$ investigated the mechanism of cracks in LDED and reduced the sensitivity of cracks by reducing heat input. One team at Mississippi State University ${ }^{103-106}$ proposed the layer-wise 
Wang et al.: Review on adaptive control of laser-directed energy deposition

Table 3 References regarding the control of LDED.

\begin{tabular}{|c|c|c|}
\hline Control objects & Parameters & References \\
\hline \multirow[t]{3}{*}{ Height } & Laser power and scanning speed & $92-95,99$, and 111 \\
\hline & Feeding rate & 112 and 113 \\
\hline & Scanning speed & 16,17, and 114 \\
\hline \multirow[t]{2}{*}{ Width } & Laser power and scanning speed & 65 \\
\hline & Spot diameter and laser power & 92 and 111 \\
\hline \multirow[t]{2}{*}{ Pool size } & Laser power and scanning speed & 93-95 and 99 \\
\hline & Laser speed & 51 \\
\hline \multirow[t]{3}{*}{ Shape } & Laser power & 51 \\
\hline & Preheating & $115-117$ \\
\hline & Scanning paths & 118 and 119 \\
\hline Cracks & Synchronized cooling, laser power, and scanning speed & 85 \\
\hline Porosities & Magnetic field & 120 \\
\hline \multirow[t]{4}{*}{ Microstructure } & Scanning speed & 45 \\
\hline & Laser power and scanning speed & $107-109$ \\
\hline & Energy density & 110 \\
\hline & Dwell time & 11 \\
\hline
\end{tabular}

processing method of multilinear principal component analysis, which can extract lowdimensional features of the molten pool to detect abnormalities in the process. The relation between molten pool morphology and microstructure anomalies was established by a machine learning framework, and the appearance of pores was predicted by a simulation model. This work considered spatial distribution of defects and proposed correction actions based on costs.

The microstructure, which is affected by temperature signatures, is related to the properties of the deposited parts, and the control of the microstructure can effectively reduce the complex postprocessing. Gockel et al. ${ }^{107-109}$ determined the proportional relation between the width of the grain and the molten pool. The size and shape of the molten pool were stable, and the solidified structure was controlled indirectly by controlling the laser power and scanning speed. Moreover, Zhang et al. ${ }^{110}$ controlled the shape of the beta grain in a relatively narrow processing parameter window by adjusting the energy density. At present, several studies have proven that effective control of the cooling rate can improve the microstructure in LDED. Farshidianfar et al. ${ }^{45}$ adjusted the scanning speed to keep the cooling rate near an ideal point and ensured the stability of the microstructure. Akbari and Kovacevic ${ }^{11}$ obtained the empirical correlation between the molten pool area and cooling rate, and controlled the final solidification structure scale by maintaining the molten pool size in real time.

In the current research, most scholars have controlled geometric accuracy, but the control of defects and microstructures is difficult; thus, the mechanical properties of parts cannot be guaranteed. Therefore, more attention needs to be paid to monitoring and control of defects and microstructure to obtain high-quality products. Table 3 shows references related to the control of LDED.

\section{Discussion}

The development of LDED is of great significance to solve the manufacturing bottleneck of several complex components. However, many shortcomings remain in the current ACT of LDED, restricting its applications. 
Compared with acquired process signatures, several important types of data, such as the microstructure and internal defects in LDED, are difficult to obtain by online monitoring. Meanwhile, traditional equipment is easily disturbed by the external environment. Therefore, more advanced equipment and technologies, such as high-speed x-ray imaging, spatialresolution acoustic spectrum, and LIBS must be applied to obtain the key information.

The relation between composite parameters (such as line energy and mass energy) and qualities remains unclear, which makes establishing an MIMO control model difficult. Therefore, a more explicit, comprehensive relation between parameters and qualities is needed. In addition, LDED initially achieved the manufacturing of precise, complex construction, but the control of stress, strain, and distortion is mostly based on experience and lacks scientific guidance at present. Establishing a large-scale, efficient simulation model of thermal, microstructure, stress, and strain to prediction qualities effectively will be one of the most important goals.

Furthermore, the ACT of LDED has made some progress, but defects such as porosity and cracks are difficult to overcome just by ACT. Several studies employed other auxiliary methods, such as using external magnetic field to control internal pores ${ }^{120}$ and using heat treatment ${ }^{85,121}$ or adding alloy components ${ }^{85}$ to inhibit cracks. Moreover, adaptive control in LDED has difficulty meeting the final industrial requirements, while the combination of AM and subtractive manufacturing will be an important way to solve this problem. Finally, the combination of digital twin and LDED will be another direction of development because LDED is a full digital manufacturing process. Each product in LDED is a natural digital twin that contains full life cycle information, and product quality can be continuously improved through online upgrades of process data and optimization algorithms.

\section{Conclusion}

LDED is a fast, low-cost, and sustainable manufacturing technique, but the quality and repeatability of parts have hindered its spread. Scholars have explored ACT in LDED to improve the stability of parts. In recent years, obtaining monitoring signatures in LDED accurately and timely, which promotes the development of the ACT in LDED, has been possible with the application of advanced monitoring equipment and processing algorithms.

This paper comprehensively reviews the application of ACT in LDED from three aspects: process monitoring; relations of process parameters, signatures, and product qualities; and adaptive control methods. Signatures such as geometry and temperature in the process can be obtained by monitoring equipment. Then, qualities such as geometric accuracy and mechanical properties can be predicted according to these signatures. Finally, product qualities will be improved by different adaptive control methods in LDED. This paper also discusses existing problems and development directions in the ACT of LDED and should enable researchers to obtain a clear vision of the research status.

\section{Acknowledgments}

This work was supported by the Liaoning Provincial Natural Science Foundation of China (Grant No. 20180520020), the Fundamental Research Funds for the Central Universities (Grant No. DUT20JC19), the Key Research and Development Plan of Ningxia (Grant No. 2018BDE02045), the Talent Project of Revitalizing Liaoning (Grant No. XLYC1802106), the Science and Technology Innovation Fund of Dalian (Grant No. 2020JJ26GX040), and Collaborative Innovation Center of Major Machine Manufacturing in Liaoning.

\section{References}

1. W. E. Frazier, "Metal additive manufacturing: a review," J. Mater. Eng. Perform. 23(6), 1917-1928 (2014).

2. D. D. Gu et al., "Laser additive manufacturing of metallic components: materials, processes and mechanisms," Int. Mater. Rev. 57(3), 133-164 (2013). 
3. D. Herzog et al., "Additive manufacturing of metals," Acta Mater. 117, 371-392 (2016).

4. S. M. Thompson et al., "An overview of direct laser deposition for additive manufacturing; part I: transport phenomena, modeling and diagnostics," Addit. Manuf. 8, 36-62 (2015).

5. N. Shamsaei et al., "An overview of direct laser deposition for additive manufacturing; part II: mechanical behavior, process parameter optimization and control," Addit. Manuf. 8, 12-35 (2015).

6. G. Tapia and A. Elwany, "A review on process monitoring and control in metal-based additive manufacturing," J. Manuf. Sci. Eng. 136(6), 060801 (2014).

7. J. Xiong and G. Zhang, "Online measurement of bead geometry in GMAW-based additive manufacturing using passive vision," Meas. Sci. Technol. 24(11), 5103 (2013).

8. J. Zhao et al., "Effects of metal-vapor jet force on the physical behavior of melting wire transfer in electron beam additive manufacturing," J. Mater. Process. Technol. 220, 243-250 (2015).

9. J. T. Hofman et al., "A camera based feedback control strategy for the laser cladding process," J. Mater. Process. Technol. 212(11), 2455-2462 (2012).

10. S. Moralejo et al., "A feedforward controller for tuning laser cladding melt pool geometry in real time," Int. J. Adv. Manuf. Technol. 89(1-4), 821-831 (2016).

11. M. Akbari and R. Kovacevic, "An investigation on mechanical and microstructural properties of 316LSi parts fabricated by a robotized laser/wire direct metal deposition system," Addit. Manuf. 23, 487-497 (2018).

12. M. Gharbi et al., "Influence of a pulsed laser regime on surface finish induced by the direct metal deposition process on a Ti64 alloy," J. Mater. Process. Technol. 214(2), 485-495 (2014).

13. U. Thombansen and M. Ungers, "Illumination for process observation in laser material processing," in 8th Int. Conf. Laser Assist. Net Shape Eng., M. Schmidt, F. Vollertsen, and M. Merklein, Eds., Elsevier Science Bv, Fürth, Germany, pp. 1286-1296 (2014).

14. C. Doumanidis and Y. M. Kwak, "Multivariable adaptive control of the bead profile geometry in gas metal arc welding with thermal scanning," Int. J. Press. Vessels Pip. 79(4), 251-262 (2002).

15. L. Song et al., "Control of melt pool temperature and deposition height during direct metal deposition process," Int. J. Adv. Manuf. Technol. 58(1-4), 247-256 (2011).

16. I. Garmendia et al., "Development of an intra-layer adaptive toolpath generation control procedure in the laser metal wire deposition process," Materials 12(3), 352 (2019).

17. I. Garmendia et al., "In-process height control during laser metal deposition based on structured light 3D scanning," in 19th Cirp Conf. Electro Phys. and Chem. Mach., A. Aranzabe, X. Maidagan, and J. A. Sanchez, Eds., Elsevier Science Bv, Bilbao, Spain, pp. 375-380 (2018).

18. S. Tang, G. Wang, and H. Zhang, "In situ 3D monitoring and control of geometric signatures in wire and arc additive manufacturing," Surf. Topogr. Metrol. Prop. 7(2), 025013 (2019).

19. S. Chang et al., "Online measurement of deposit surface in electron beam freeform fabrication," Sensors (Basel) 19(18), 4001 (2019).

20. V. Ocelik, J. Bosgra, and J. T. M. de Hosson, "In-situ strain observation in high power laser cladding," Surf. Coat. Technol. 203(20-21), 3189-3196 (2009).

21. M. Biegler, B. Graf, and M. Rethmeier, "In-situ distortions in LMD additive manufacturing walls can be measured with digital image correlation and predicted using numerical simulations," Addit. Manuf. 20, 101-110 (2018).

22. J. L. Bartlett et al., "Revealing mechanisms of residual stress development in additive manufacturing via digital image correlation," Addit. Manuf. 22, 1-12 (2018).

23. R. Xie et al., "In-situ observation and numerical simulation on the transient strain and distortion prediction during additive manufacturing," J. Manuf. Processes 38, 494-501 (2019).

24. Z. Yan et al., "Effect of thermal characteristics on distortion in laser cladding of AISI 316L," J. Manuf. Processes 44, 309-318 (2019). 
25. S. Morville et al., " $2 \mathrm{D}$ longitudinal modeling of heat transfer and fluid flow during multilayered direct laser metal deposition process," J. Laser Appl. 24(3), 032008 (2012).

26. Z. Yan et al., "Review on thermal analysis in laser-based additive manufacturing," Opt. Laser Technol. 106, 427-441 (2018).

27. E. R. Denlinger et al., "Effect of inter-layer dwell time on distortion and residual stress in additive manufacturing of titanium and nickel alloys," J. Mater. Process. Technol. 215, 123-131 (2015).

28. A. Segerstark, J. Andersson, and L.-E. Svensson, "Evaluation of a temperature measurement method developed for laser metal deposition," Sci. Technol. Weld. Joining 22(1), 1-6 (2016).

29. J. Hartmann, "High-temperature measurement techniques for the application in photometry, radiometry and thermometry," Phys. Rep. 469(5-6), 205-269 (2009).

30. D. De Baere et al., "Spectroscopic monitoring and melt pool temperature estimation during the laser metal deposition process," J. Laser Appl. 28(2), 022303 (2016).

31. W. Devesse et al., "Model-based temperature feedback control of laser cladding using high-resolution hyperspectral imaging," IEEE/ASME Trans. Mechatron. 22(6), 2714-2722 (2017).

32. W. Devesse et al., "Hardware-in-the-loop control of additive manufacturing processes using temperature feedback," J. Laser Appl. 28(2), 022302 (2016).

33. G. Bi et al., "Identification and qualification of temperature signal for monitoring and control in laser cladding," Opt. Lasers Eng. 44(12), 1348-1359 (2006).

34. G. Bi et al., "Characterization of the process control for the direct laser metallic powder deposition," Surf. Coat. Technol. 201(6), 2676-2683 (2006).

35. M. Doubenskaia, P. Bertrand, and I. Smurov, "Optical monitoring of Nd:YAG laser cladding," Thin Solid Films 453-454, 477-485 (2004).

36. G. Bi, C. N. Sun, and A. Gasser, "Study on influential factors for process monitoring and control in laser aided additive manufacturing," J. Mater. Process. Technol. 213(3), 463-468 (2013).

37. G. Muvvala, D. Patra Karmakar, and A. K. Nath, "Online monitoring of thermo-cycles and its correlation with microstructure in laser cladding of nickel based super alloy," Opt. Lasers Eng. 88, 139-152 (2017).

38. D. Salehi and M. Brandt, "Melt pool temperature control using LabVIEW in Nd:YAG laser blown powder cladding process," Int. J. Adv. Manuf. Technol. 29(3-4), 273-278 (2005).

39. L. Tang and R. G. Landers, "Melt pool temperature control for laser metal deposition processes-part I: online temperature control," J. Manuf. Sci. Eng. 132(1), 011010 (2010).

40. L. Tang and R. G. Landers, "Melt pool temperature control for laser metal deposition processes-part II: layer-to-layer temperature control," J. Manuf. Sci. Eng. 132(1), 011011 (2010).

41. L. Song and J. Mazumder, "Feedback control of melt pool temperature during laser cladding process," IEEE Trans. Control Syst. Technol. 19(6), 1349-1356 (2011).

42. M. Pavlov, D. Novichenko, and M. Doubenskaia, "Optical diagnostics of deposition of metal matrix composites by laser cladding," in Lasers in Manuf. 2011: Proc. Sixth Int. WLT Conf. Lasers in Manuf., M. Schmidt et al., Eds., Vol. 12, Pt. A, Elsevier Science Bv, Munich, Germany, pp. 674-682 (2011).

43. M. Doubenskaia et al., "Optical monitoring in elaboration of metal matrix composites by direct metal deposition," in Laser Assisted Net Shape Eng., M. Schmidt, F. Vollertsen, and M. Geiger, Eds., Elsevier Science Bv, Fürth, Germany, pp. 767-775 (2012).

44. D. Yang, G. Wang, and G. Zhang, "Thermal analysis for single-pass multi-layer GMAW based additive manufacturing using infrared thermography," J. Mater. Process. Technol. 244, 215-224 (2017).

45. M. H. Farshidianfar, A. Khajepour, and A. Gerlich, "Real-time control of microstructure in laser additive manufacturing," Int. J. Adv. Manuf. Technol. 82(5-8), 1173-1186 (2015).

46. M. H. Farshidianfar, A. Khajepour, and A. P. Gerlich, "Effect of real-time cooling rate on microstructure in laser additive manufacturing," J. Mater. Process. Technol. 231 468-478 (2016). 
47. I. Smurov et al., "Optical monitoring in laser cladding of Ti6Al4V," J. Therm. Spray Technol. 21(6), 1357-1362 (2012).

48. S. Liu, P. Farahmand, and R. Kovacevic, "Optical monitoring of high power direct diode laser cladding," Opt. Laser Technol. 64, 363-376 (2014).

49. G. J. Marshall, S. M. Thompson, and N. Shamsaei, "Data indicating temperature response of Ti-6Al-4V thin-walled structure during its additive manufacture via laser engineered net shaping," Data Brief 7, 697-703 (2016).

50. G. J. Marshall et al., "Understanding the microstructure formation of Ti-6Al-4V during direct laser deposition via in-situ thermal monitoring," JOM 68(3), 778-790 (2016).

51. M. Miyagi, T. Tsukamoto, and H. Kawanaka, "Adaptive shape control of laser-deposited metal structures by adjusting weld pool size," J. Laser Appl. 26(3), 032003 (2014).

52. Y. Ding, J. Warton, and R. Kovacevic, "Development of sensing and control system for robotized laser-based direct metal addition system," Addit. Manuf. 10, 24-35 (2016).

53. F. Wang et al., "Online study of cracks during laser cladding process based on acoustic emission technique and finite element analysis," Appl. Surf. Sci. 255(5), 3267-3275 (2008).

54. H. Gaja and F. Liou, "Defects monitoring of laser metal deposition using acoustic emission sensor," Int. J. Adv. Manuf. Technol. 90(1-4), 561-574 (2016).

55. M. Vargas et al., "X-ray phase contrast imaging of additive manufactured structures using a laser wakefield accelerator," Plasma Phys. Controlled Fusion 61(5) (2019).

56. S. J. Wolff et al., "In-situ high-speed x-ray imaging of piezo-driven directed energy deposition additive manufacturing," Sci. Rep. 9(1), 962 (2019).

57. S. D. Sharples, M. Clark, and M. G. Somekh, "Spatially resolved acoustic spectroscopy for fast noncontact imaging of material microstructure," Opt. Express 14(22), 10435-10440 (2006).

58. M. Hirsch et al., "Meso-scale defect evaluation of selective laser melting using spatially resolved acoustic spectroscopy," Proc. Math. Phys. Eng. Sci. 473(2205), 20170194 (2017).

59. R. Patel et al., "Imaging material texture of as-deposited selective laser melted parts using spatially resolved acoustic spectroscopy," Appl. Sci. 8(10), 1991 (2018).

60. R. J. Smith et al., "Spatially resolved acoustic spectroscopy for selective laser melting," J. Mater. Process. Technol. 236, 93-102 (2016).

61. V. N. Lednev et al., "In situ multi-elemental analysis by laser induced breakdown spectroscopy in additive manufacturing," Addit. Manuf. 25, 64-70 (2019).

62. D. W. Hahn and N. Omenetto, "Laser-induced breakdown spectroscopy (LIBS), part II: review of instrumental and methodological approaches to material analysis and applications to different fields," Appl. Spectrosc. 66(4), 347-419 (2012).

63. L. Song and J. Mazumder, "Real time Cr measurement using optical emission spectroscopy during direct metal deposition process," IEEE Sens. J. 12(5), 958-964 (2012).

64. V. Ocelík et al., "Thick Co-based coating on cast iron by side laser cladding: analysis of processing conditions and coating properties," Surf. Coat. Technol. 201(12), 5875-5883 (2007).

65. H. Qi, M. Azer, and P. Singh, "Adaptive toolpath deposition method for laser net shape manufacturing and repair of turbine compressor airfoils," Int. J. Adv. Manuf. Technol. 48(1-4), 121-131 (2009).

66. R. Parekh, R. K. Buddu, and R. I. Patel, "Multiphysics simulation of laser cladding process to study the effect of process parameters on clad geometry," Procedia Technol. 23, 529-536 (2016).

67. P. Sreeraj and T. Kannan, "Modelling and prediction of stainless steel clad bead geometry deposited by GMAW using regression and artificial neural network models," Adv. Mech. Eng. 2012, 237379 (2012).

68. K. Zhang, X. M. Zhang, and W. J. Lu, "Influences of processing parameters on dilution ratio of laser cladding layer during laser metal deposition shaping," in Chemical Engineering and Material Properties II, B. Li, Ed., pp. 785-789, Trans Tech Publications Ltd., Taiyuan, China (2012).

69. J. T. Hofman et al., "FEM modeling and experimental verification for dilution control in laser cladding," J. Mater. Process. Technol. 211(2), 187-196 (2011). 
70. W. Xi et al., "Geometry and dilution rate analysis and prediction of laser cladding," Int. J. Adv. Manuf. Technol. 103(9-12), 4695-4702 (2019).

71. Q. Tang et al., "A three dimensional transient model for heat transfer and fluid flow of weld pool during electron beam freeform fabrication of Ti6-Al-4-V alloy," Int. J. Heat Mass Transfer 78, 203-215 (2014).

72. Y. Huang et al., "Rapid prediction of real-time thermal characteristics, solidification parameters and microstructure in laser directed energy deposition (powder-fed additive manufacturing)," J. Mater. Process. Technol. 274, 116286 (2019).

73. T. Mukherjee et al., "Mitigation of thermal distortion during additive manufacturing," Scr. Mater. 127, 79-83 (2017).

74. T. Mukherjee, W. Zhang, and T. DebRoy, "An improved prediction of residual stresses and distortion in additive manufacturing," Comput. Mater. Sci. 126, 360-372 (2017).

75. R. Xie et al., "Development of efficient distortion prediction numerical method for laser additive manufactured parts," J. Laser Appl. 31(2), 022314 (2019).

76. M. Nabhani, R. S. Razavi, and M. Barekat, "An empirical-statistical model for laser cladding of Ti-6Al-4V powder on Ti-6Al-4V substrate," Opt. Laser Technol. 100, 265-271 (2018).

77. J. P. Davim, C. Oliveira, and A. Cardoso, "Laser cladding: an experimental study of geometric form and hardness of coating using statistical analysis," Proc. Inst. Mech. Eng. Pt. B: J. Eng. Manuf. 220(9), 1549-1554 (2006).

78. H. Zhang et al., "Analytical modeling and experimental investigation of laser clad geometry," Opt. Eng. 56(9), 096104 (2017).

79. F. Vásquez, J. A. Ramos-Grez, and M. Walczak, "Multiphysics simulation of lasermaterial interaction during laser powder depositon," Int. J. Adv. Manuf. Technol. 59(9-12), 1037-1045 (2011).

80. J. Li et al., "An extended lumped-parameter model of melt-pool geometry to predict part height for directed energy deposition," J. Manuf. Sci. Eng. 139(9), 091016 (2017).

81. Q. Wang et al., "Physics-based multivariable modeling and feedback linearization control of melt-pool geometry and temperature in directed energy deposition," J. Manuf. Sci. Eng. 139(2), 021013 (2017).

82. S. S. Chakraborty and S. Dutta, "Estimation of dilution in laser cladding based on energy balance approach using regression analysis," Sadhana 44(6), 150 (2019).

83. F. Du et al., "Dimensional characteristics of Ti-6Al-4V thin-walled parts prepared by wire-based multi-laser additive manufacturing in vacuum," Rapid Prototyping $J$ 25(5), 849-856 (2019).

84. G. Zhu et al., "The influence of laser and powder defocusing characteristics on the surface quality in laser direct metal deposition," Opt. Laser Technol. 44(2), 349-356 (2012).

85. Z. Chen et al., "Methods of crack control for Inconel 738 laser cladding layer," Appl. Laser 33(1), 7-13 (2013)

86. T. Amine, J. W. Newkirk, and F. Liou, "Investigation of effect of process parameters on multilayer builds by direct metal deposition," Appl. Therm. Eng. 73(1), 500-511 (2014).

87. A. Fathi et al., "Clad height control in laser solid freeform fabrication using a feedforward PID controller," Int. J. Adv. Manuf. Technol. 35(3-4), 280-292 (2006).

88. J. I. Arrizubieta et al., "Instantaneous powder flux regulation system for laser metal deposition," J. Manuf. Processes 29, 242-251 (2017).

89. P. M. Sammons et al., "Repetitive process control of additive manufacturing with application to laser metal deposition," IEEE Trans. Control Syst. Technol. 27(2), 566-575 (2019).

90. P. M. Sammons, D. A. Bristow, and R. G. Landers, "Two-dimensional modeling and system identification of the laser metal deposition process," J. Dyn. Syst. Meas. Control 141(2), 10 (2019).

91. S. Liu et al., "Study of a hollow laser beam for cladding," Int. J. Adv. Manuf. Technol. 73(1-4), 147-159 (2014).

92. T. Shi et al., "Closed-loop control of variable width deposition in laser metal deposition," Int. J. Adv. Manuf. Technol. 97(9-12), 4167-4178 (2018). 
93. X. Q. Cao and B. Ayalew, "Multivariable predictive control of laser-aided powder deposition processes," in Am. Control Conf., IEEE, Chicago, Illinois, pp. 3625-3630 (2015).

94. X. Q. Cao and B. Ayalew, "Control-oriented MIMO modeling of laser-aided powder deposition processes," in Am. Control Conf., IEEE, Chicago, Illinois, pp. 3637-3642 (2015).

95. X. Cao and B. Ayalew, "Robust multivariable predictive control for laser-aided powder deposition processes," J. Franklin Inst. 356(5), 2505-2529 (2019).

96. L. Wang et al., "Optimization of the LENS ${ }^{\circledR}$ process for steady molten pool size," Mat. Sci. Eng. A-Struct. 474(1-2), 148-156 (2008).

97. J. Rodriguez-Araujo et al., "Industrial laser cladding systems: FPGA-based adaptive control," IEEE Ind. Electron. Mag. 6(4), 35-46 (2012).

98. M. M. Anzehaee and M. Haeri, "Estimation and control of droplet size and frequency in projected spray mode of a gas metal arc welding (GMAW) process," ISA Trans. 50(3), 409-418 (2011).

99. X. Cao and B. Ayalew, "Partial differential equation-based multivariable control input optimization for laser-aided powder deposition processes," J. Manuf. Sci. Eng. 138(3), 031001 (2015).

100. W. Devesse, D. De Baere, and P. Guillaume, "Design of a model-based controller with temperature feedback for laser cladding," in 8th Int. Conf. Laser Assist. Net Shape Eng., M. Schmidt, F. Vollertsen, and M. Merklein, Eds., Elsevier Science Bv, Fürth, Germany, pp. 211-219 (2014).

101. W. Devesse, D. De Baere, and P. Guillaume, "The isotherm migration method in spherical coordinates with a moving heat source," Int. J. Heat Mass Transfer 75, 726-735 (2014).

102. J. C. Heigel, P. Michaleris, and T. A. Palmer, "In situ monitoring and characterization of distortion during laser cladding of Inconel ${ }^{\circledR} 625$, , J. Mater. Process. Technol. 220, 135-145 (2015).

103. M. Khanzadeh et al., "Dual process monitoring of metal-based additive manufacturing using tensor decomposition of thermal image streams," Addit. Manuf. 23, 443-456 (2018).

104. M. Khanzadeh et al., "Porosity prediction: supervised-learning of thermal history for direct laser deposition," J. Manuf. Syst. 47, 69-82 (2018).

105. S. H. Seifi et al., "Layer-wise modeling and anomaly detection for laser-based additive manufacturing," J. Manuf. Sci. Eng. 141(8), 081013 (2019).

106. R. Jafari-Marandi et al., "From in-situ monitoring toward high-throughput process control: cost-driven decision-making framework for laser-based additive manufacturing," J. Manuf. Syst. 51, 29-41 (2019).

107. J. Gockel et al., "Trends in solidification grain size and morphology for additive manufacturing of Ti-6Al-4V," JOM 69(12), 2706-2710 (2017).

108. J. Gockel et al., "Integrated melt pool and microstructure control for Ti-6Al-4V thin wall additive manufacturing," Mater. Sci. Technol. 31(8), 912-916 (2014).

109. J. Gockel, J. Beuth, and K. Taminger, "Integrated control of solidification microstructure and melt pool dimensions in electron beam wire feed additive manufacturing of Ti-6Al4V," Addit. Manuf. 1-4, 119-126 (2014).

110. Q. Zhang et al., "Grain morphology control and texture characterization of laser solid formed Ti6Al2Sn2Zr3Mo1.5Cr2Nb titanium alloy," J. Mater. Process. Technol. 238, 202-211 (2016).

111. T. Shi et al., "Laser metal deposition with spatial variable orientation based on hollow-laser beam with internal powder feeding technology," Opt. Laser Technol. 88, 234-241 (2017).

112. A. Heralić, A.-K. Christiansson, and B. Lennartson, "Height control of laser metal-wire deposition based on iterative learning control and 3D scanning," Opt. Lasers Eng. 50(9), 1230-1241 (2012).

113. M. Zeinali and A. Khajepour, "Development of an adaptive fuzzy logic-based inverse dynamic model for laser cladding process," Eng. Appl. Artif. Intell. 23(8), 1408-1419 (2010).

114. I. Garmendia et al., "Structured light-based height control for laser metal deposition," J. Manuf. Processes 42, 20-27 (2019).

115. N. W. Klingbeil et al., "Residual stress-induced warping in direct metal solid freeform fabrication," Int. J. Mech. Sci. 44(1), 57-77 (2002). 
116. J. Cao, M. A. Gharghouri, and P. Nash, "Finite-element analysis and experimental validation of thermal residual stress and distortion in electron beam additive manufactured Ti-6Al-4V build plates," J. Mater. Process. Technol. 237, 409-419 (2016).

117. D. J. Corbin et al., "Effect of Substrate thickness and preheating on the distortion of laser deposited Ti-6Al-4V," J. Manuf. Sci. Eng. 140(6), 061009 (2018).

118. M. Gao et al., "The effect of deposition patterns on the deformation of substrates during direct laser fabrication," J. Eng. Mater 135(3), 034502 (2013).

119. H. Yan et al., "Stress and deformation evaluation of the subarea scanning effect in direct laser-deposited Ti-6Al-4V," Int. J. Adv. Manuf. Technol. 97(1-4), 915-926 (2018).

120. N. Zhang et al., "Effect of electric-magnetic compound field on the pore distribution in laser cladding process," Opt. Laser Technol. 108, 247-254 (2018).

121. X. Xing et al., "Microstructure optimization and cracking control of additive manufactured bainite steel by gas metal arc welding technology," J. Mater. Eng. Perform. 28(8), 5138-5145 (2019).

Weiwei Liu is an associate professor at the School of Mechanical Engineering, Dalian University of Technology. His research focuses on additive manufacturing, LDED, machine vision, and intelligent control system.

Biographies of the other authors are not available. 